\title{
EL ENVEJECIMIENTO DE LA POBLACIÓN \\ Y LA ENFERMEDAD DE ALZHEIMER, FUTUROS RETOS PARA ENFERMERÍA
}

Biól. Victor Valverde Molina*

* Profesor de la materia de Ecología y Salud, ENEO, UNAM.

\section{Restument}

El envejecimiento continuo de la población mundial exige que los gobiemos y la sociedad civil trabajen de manera conjunta, para evitar que los servicios de salud se saturen.

La enfermedad de Alzheimer gana terreno entre los adultos mayores y se caracteriza por alteraciones en la esfera conductual y psicológica que tiene repercusiones a nivel familiar y por ende a nivel social. Los apoyos farmacológicos existentes frenan temporalmente el avance de la enfermedad en las primeras etapas, por lo que se enfatiza el trabajo hacia las causas y los factores de riesgo.

El personal de Enfermeria apoyado en un trabajo multidisciplinario podria ser punta de lanza para contener este tipo de demencia. Debido a su eminente vocación educativa que inicia con la prevención y culmina en la atención directa đel paciente.

PALABRAS CLAVE: Alzheimer, Enfermeria, demencia, enfermedades neurodegenerativas, envejecimiento

\section{Abstract}

Continuous ageing on mundial population demands that government and civil society work together to avoid saturation of health services.

Alzheimer illness grows rapidly among adults and is characterized by alteration in conductual and psychological behaviour repercution in familiar and social levels. Existing pharmacological aid diminish temporally this illness evolution in its first phases, that's why work and effort are fawsed on its couses and risks.

Nursery personnel, based in a multidiscplinary work could be the bose to work with this type of insane illness. Due to its eminent educative vocation that starts with prevention and ends with direct treatment of the patients.

KEY WORDS: Alzheimer, Nursery, dementia, neurodegenerative disease, aging.

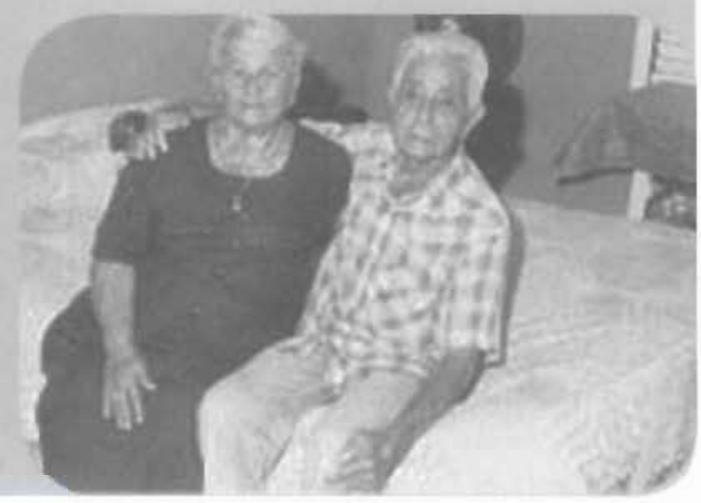




\section{INTRODUCCIÓN}

En estos momentos nos sentimos satisfechos con los logros alcanzadosi a nivel mundial en cuanto a las mejoras constantes en la esperanza de vida, así como de las tasas de fertilidad decrecientes y un aumento de la longevidad. El "envejecimiento" continuo de la población mundial requiere de un nuevo abordaje a escala mundial, ya que definitivamente tendrá muy particulares exigencias a nivel económico y social en todos los países.

La Organización Mundial de la Salud exhorta a los países a enfrentar el envejecimiento implementando políticas y programas de "envejecimiento activo" que mejoren la salud, la participación y la seguridad de los ciudadanos de mayor edad. En el entendido de que los gobiernos, las organizaciones internacionales y la sociedad civil trabajen de manera conjunta. De lo contrario las consecuencias serían desastrosas para la salud pública debido a la posibilidad de que se sobrecargue los servicios existentes y de que con ello se establezca una competencia intergeneracional por los recursos'.

"En nuestra región el número de personas de 60 años de edad y mayores es actualmente de 91 millones. Una tasa prevista de crecimiento de 3,5\% en esta población durante los dos primeros decenios del siglo XXI hará crecer esa cifra a 194 millones, y alcanzará los 292 millones a mediados del siglo. Actualmente, las personas adultas mayores representan 8\% de la población total en América Latina y el Caribe, y 16\% en Canadá y los Estados Unidos. Para el año 2025, 14\% de la población de América Latina y el Caribe tendrá 60 años de edad o más" 2.

El personal de Enfermería se caracteriza por ser el primer contacto con la población demandante, por ser el puente con las demás especialidades y áreas de la salud. Situación privilegiada que le permitiría anticiparse a la demanda de servicios de salud a través del fortalecimiento de los modelos de atención así como la implementación de programas de acción para la atención integral de los pacientes. Es decir, los profesionales de Enfermería tendrán que desarrollar programas de educación para la salud bajo un enfoque preventivo y no meramente curativo. Implementando estrategias de atención que sean congruentes con las verdaderas necesidades de los adultos mayores ${ }^{3}$

\section{VEJEZ: ¿SINÓNIMO DE DECADENCIA?}

Se nos olvida que la vejez es un proceso como lo es la infancia, la juventud y la adultez. Es la etapa final de nuestro recorrido biológico que como tal marca limitacio- nes en el sistema locomotor, cardiovascular y los órganos de los sentidos. Empero como entes sociales la actividad productiva y la interacción social, son sustituidas en algunos casos por la jubilación. La pérdida de algunos seres queridos y la reducción del grupo de pertenencia complican el panorama.

En resumen, estamos de cara a una situación que se antoja compleja a todos los niveles, ya que las enfermedades crónico-degenerativas comienzan a colocarse dentro de los primeros lugares $y$ arrastran consigo una serie de secuelas incapacitantes e invalidantes. De las cuales hemos seleccionado por $5 u$ rápida emergencia a la llamada Enfermedad de Alzheimer (EA O DA).

El estudio metódico de la EA en Norteamérica y el bloque europeo (EURODEM, eura-demencia) nos muestra cifras dignas de tener en cuenta. Por ejemplo, en EE. UU. Se calcula que existen 4 millones de candidatos y que cada 5 años se duplica la cifra a partir de los 65 años. Así tenemos que al año se detectan 360000 casos, número que aumentará conforme la población envejezca. Si hacemos la traducción a términos monetarios estariamos hablando de aproximadamente cien millones de dólares`. En la Unión Europea se calcula que las demencias afectan a 3386000 individuos y que surgen 824000 casos nuevos cada años.

En México la EA, está subdiagnosticada por la falta de un estudio epidemiológico formal y por el momento se tiene un estimado de entre 300 mil a 600 mil mexicanos. Cifra que puede ser mayor si consideramos que el grueso de la población asocia la sintomatología de EA con el proceso natural del envejecimiento ${ }^{5}$

\section{ENFERMEDAD DE ALZHEIMER.}

Antes de comenzar con el tratamiento de la EA es pertinente aclarar que el Alzheimer es un. sindrome y no necesariamente una consecuencia del proceso normal de la vejez.

La EA se clasifica dentro de los llamados trastornos neurodegenerativos que inicia con pérdida de memoria, al que se van sumando alteraciones de otras funciones mentales. En conjunto la sintomatologia impacta de manera contundente a los familiares, a los cuidadores, a la sociedad y culmina con el ingreso institucional en la mayoría de los casos que llegan a una etapa avanzada.

Al igual que la EA existen otras demencias como la demencia vascular, la demencia, frontal, la enfermedad por cuerpos de Lewy, por citar las más frecuentes y van a cur- 
sar con deterioro de la función cognitiva, aparición de dependencia funcional, alteraciones del comportamiento más o menos graves y una progresiva necesidad de cuidados? Por tanto, es importante discriminar una de otra. Labor nada sencilla que recae en la aplicación de los instrumentos y estudios del especialista en Neuropsicología.

\section{NEUROPSICOLOGÍA.}

La clínica neuropsicológica cuenta con diferentes pruebas a través de las cuales se evalúa el comportamiento cerebral. Determina de manera confiable un posible déficit cognitivo adquirido, por ejemplo, consecuencia de traumatismo encefálico, consecuencia de accidente cerebro vascular, consecuencia de enfermedades degenerativas, intoxicaciones; etc. La evaluación neuropsicológica evidencia el estado de las funciones cognitivas y las funciones ejecutivas. Resulta de gran ayuda para diferenciar entre sintomas psiquiátricos y síntomas neurológicos așí como el estado de las condiciones neurológicas, En suma, arroja información sobre la conducta cerebral para poder localizar la lesión".

La EA se encuentra dentro de las llamadas demencias que se caracterizan por la pérdida de la memoria y otras capacidades intelectuales que se diagnostica de acuerdo a los criterios utilizados en la cuarta edición del manual diagnóstico y estadístico de la asociación Americana de Psiquiatría (DSM-IV). Los sintomas nos indican el avance de la enfermedad y van desde las fases iniciales donde está afectada la memoria a corto y largo plazo así como la ubicación espacio-temporal; cambios importantes como irritabilidad, ansiedad, alteraciones. en la personalidad y depresión. Se incorporan posteriormente: alteraciones cognoscitivas: afasia (alteraciones del lenguaje), apraxia (incapacidad para realizar actividades motoras), agnosia (problemas en el reconocimiento de objetos) y alteracio-. nes en el juicio, (cuadro No. 1); aparecen comportamientos agresivos, delirios y alucinaciones?.

El diagnóstico se confirma con neuroimágenes como la resonancia magnética, tomografía de emisión de positrones o la tomografia computarizada de emisión unifónica.

\section{ETIOLOGÍA.}

La etiologia es multifactorial con diversos factores de riesgo como se muestra en el cuadro No. 2.

\section{Histopatología.}

La EA se caracteriza por la evidente relación entre las lesiones neurofibrilares y los depósitos del beta amiloide a

\section{Cuadro No. 1. Evolución de la EA.}

\section{- Primera fase}

- La persona olvida algunas cosas.

- Comienza a perder vocabulario.

- Siente dificultad para realizar actividades.

- Además momentáneamente pierde la referencia espacio-temporal.

- Se alteran aspectos de su personalidad.

- Dura aproximadamente entre 2 a 5 años

\section{- Segunda fase}

- El enfermo olvida los sucesos recientes.

- Apenas puede decir unas cuantas frases seguidas.

- Abandona sus actividades.

- Se pierde por la calle.

- Puede tener reacciones desmesuradas en su carácter.

- Dura entre 2 a 8 años

\section{- Tercera fase}

- Agnosia extrema. No conoce a nadie ni reconoce nada. Sin embargo, se da cuenta de la persona que lo atiende.

- Afasia. No habla ni entiende nada, aunque aprecia y valora el sentido del tacto.

- Apraxia. No realiza ninguna actividad. 


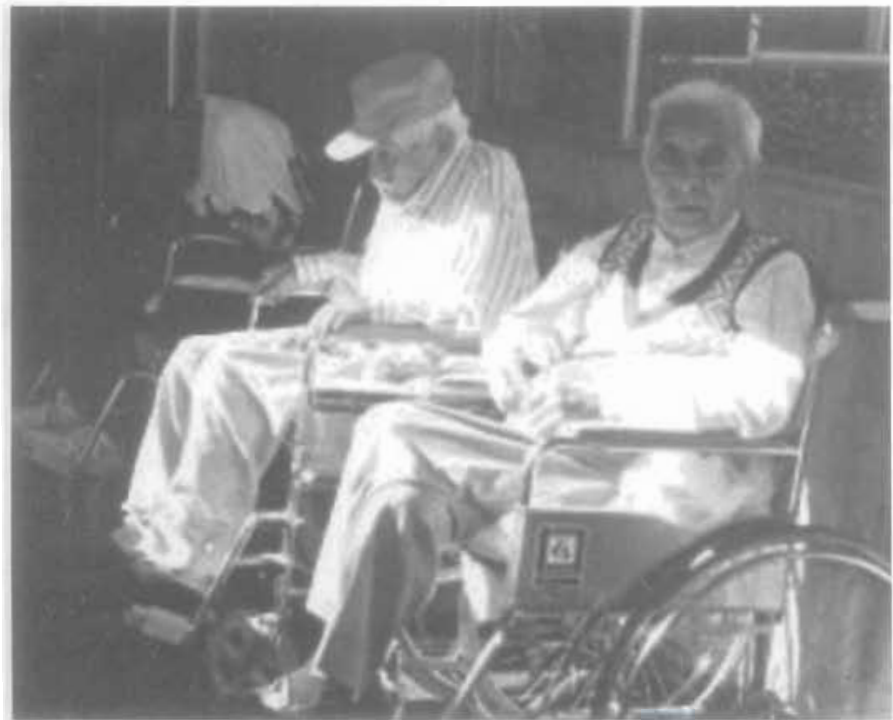

nivel hitopatológico. Las primeras se encuentran en los. cuerpos de las células neuronales (racimos neurofibrilares arreglados en filamentos helicoidales en pares) y en los procesos neuronales (filamentos neuropilares) con la proteína tau asociada al microtúbulo que interfiere con el funcionamiento normal del transporte intracelular. Los depósitos amiloides, producto de la fragmentación de una proteina denominada proteina precursora del amiloide, se encuentran alrededor y dentro de las paredes de los vasos sanguineos (angiopatia amiloide cerebral) y en parénquimas cerebrales (placas seniles). Se cree que el beta amiloide induce a un daño oxidativo en células vecinas, estimula la apoptosis (muerte celular), dificulta la perfusión, afecta los contactos sinápticos y activa la microglia ${ }^{14}$. A medida que estos cambios celulares avanzan, se pierden neuronas en la circunvolución del hipocampo, la corteza entorenal y las zonas relacionadas con la corteza cerebral!'s.

\section{TRATAMIENTO.}

En este momento se cuenta con múltiples alternativas terapéuticas farmacológicas y no farmacológicas que deben adecuarse estrictamente a la etapa por la que cursa el paciente. Para evitar efectos colaterales e indeseables sobre todo cuando se utilizan fármacos. Los medicamentos hasta ahora utilizados no pueden ser considerados los más eficientes y adolecen de muchas propiedades para poder modificar la enfermedad en su totalidad. Situación que nos reorienta de nueva cuenta hacia la parte preventiva, y por tanto, a las acciones de Enfermería en este rubro que, por ningún motivo son las únicas en las cuales pueden participar pero si las de mayor impacto a largo plazo. Acciones aparentemente sencillas como educar a la población para favorecer la consulta precoz;, motivarla y hacerla participe del tratamiento de los pacientes, permitirian no sólo optimizar tiempos sino recursos ${ }^{16}$. Hoy más que nunca el trabajo interdisciplinario para atenuar este tipo de demencia reclama la aportación del licenciado en Enfermería!?

\section{CONCLUSIONES}

La EA nos conduce hacia un abordaje terapéutico multidimensional con intervenciones farmacológicas e intervenciones no farmacológicas que en conjunto tienden a optimizar la cognición, la conducta y la función de los sujetos con demencia. Sin dejar de lado las necesidades de los cuidadores. Por tal motivo, la contención familiar, las oportunidades sociales y el nivel de información con respecto a este tipo de enfermedad (y todas las crónicodegenerativas) nos pondrán en la balanza y ejercerán un peso específico que se inclinará para dignificar o deteriorar este proceso del "envejecer".

En México requerimos formar personal de Enfermería capacitado para las enfermedades asociadas a la vejez como es la EA. Debido a que una intervención apropiada que retrase su inicio por lo menos en 5 años podría reducir a la mitad el número de pacientes para el año 2050, cifra nada modesta.

Considero que el Licenciado en Enfermería tiene en este momento una oportunidad histórica de participar de manera significativa en el adecuado tratamiento de la EA. Falta, entonces, que se definan las estrategias y áreas de trabajo. Evitando a futuro su inserción únicamente en la parte operativa.

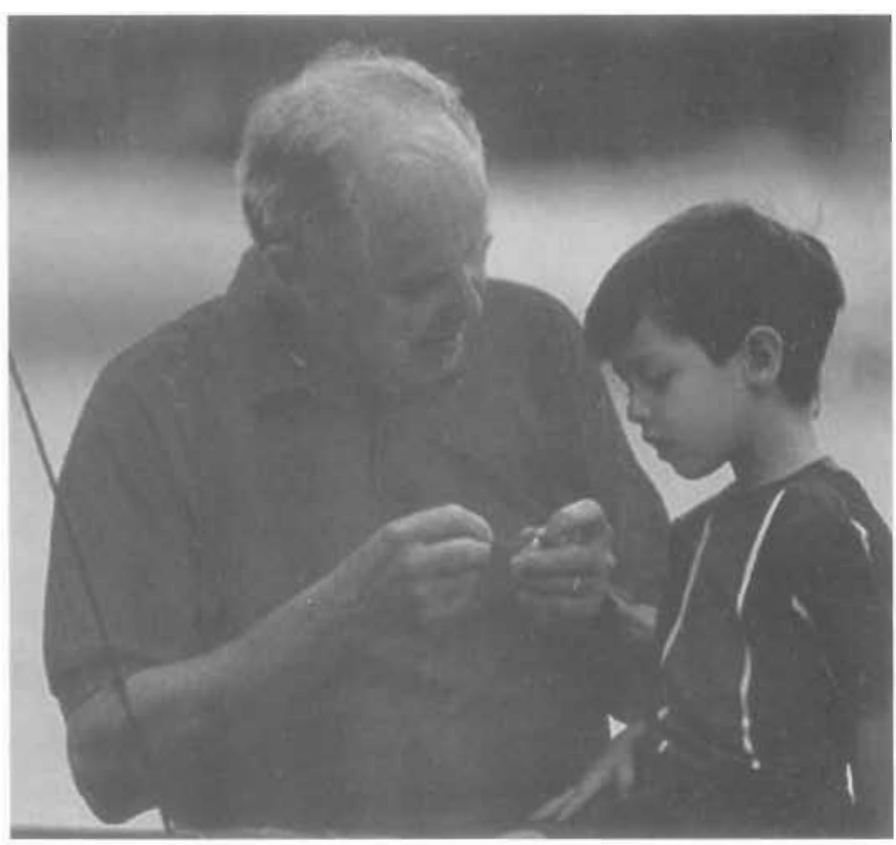


Cuadro No. 2. Factores de riesgo para la EA

\begin{tabular}{|c|c|}
\hline FACTOR & CARACTERISTICAS \\
\hline Edad & La EA aumenta del $5 \%$ al $8 \%$ en mayores de 65 años y un $30 \%$ en mayores de 85 años. \\
\hline Riesgo vascular & Hipertensión arterial, infarto de miocardio, diabetes, consumo elevado de grasas, etc. \\
\hline Daño cerebral & Traumatismos craneoencefálicos graves. \\
\hline Genético & $\begin{array}{l}\text { Cromosoma 21.- gen de la proteína (transmembranal) precursora de amiloide inductor de racimos } \\
\text { neurofibrilares y placas seniles. Aparición temprana de EA. } \\
\text { Cromosoma 19.-gen de la apolipoproteína que transporta el colesterol en sangre; se une también } \\
\text { a lesiones neurofibrilares. De aparición tardía en EA. } \\
\text { Cromosoma 14.-gen de la presenilina } 1 \text {. Aparición temprana en EA., } \\
\text { Cromosoma 1.- gen de la presenilina } 2 \text {. Aparición temprana en EA. } \\
\text { Cromosoma 12.- gen de la alfa } 2 \text { macroglobulina que inhibe la proteasa del suero. Se considera } \\
\text { dentro de las de aparición esporádica en EA }{ }^{10,11,12,13} \text {. }\end{array}$ \\
\hline
\end{tabular}

\section{REFERENCIAS BIBLIOGRÁFICAS}

1. OMS Envejecimiento activo: un marco politico. Rev Esp Geriatr Gerontol, 2002; 37(\$2):74-105

2. OPS/OMS. La salud y el envejecimiento. 36.a Sesión del subcomite de planificación y programación del comité ejecutivo. SPP36/7 (ESP.) 29 enero 2002, 19 pP.

3. Vargas-E, Luz Maria y col. Influencia de las creencias en vejez, salud y enfermedad de aduitos mayores en el uso de servicios de salud. Enfermeria Universitaria. 2005. 2 (1): 39-44.

4. Instituto Nacional de Envejecimiento (National Institute on Aging) de los Estados Unidos de América. La enfermedod de Alzheimer en el año 2000. Rev Panam Salud Publica. 2001. 10(4):268-276.

5. Launer-L; J., Hofman A. Frequency and impact of neurologic diseases in the elderly of Europe: a collaborative study of population-based cohorts. Neurology 2000; 54(supl. 5):S1-S3.

6.http://muw.familiaalzheimer.org/magazine/articulo. asp?imprime $=1$ \&id_newsletter_editorial $=43$

7. Doval, O.; Gaviria, M. Demencia Frontotemporal (Frontotemporal Dementia). Disponible en la World Wide Web: <hitp:// muw.humana.com.ve/sm/art/dft 1.doc.2002

8. Lezak, M. The practice of Neuropsychological Assessment. 3a. Ed. Oxfort University Pres. 1995. Cap. I pp. 7-16.

9. Small-G. y col. Diagnosis and treatment of Alzheimer disease and related disorders. JAMA, 1997. 278: 1363-1371.

10. Eastwood-R. y col. The challenge of the dementias. Lancet: 1996. 347: 1303-1307.
11. Hardy J. Amyloid. The presenilins and Alzheimer's disease. TINS. 1997 20: 154-158.

12. Price D, Sisodia 5, Borchelt D. Alzheimer disease- when and why? Nature Genetics, 1998. 19:314-316

13. Blacker D. y col. Alpha-2. macroglobulin is genetically associated with Alzheimer disease. Nature Genetics. 1998. 19:357-360.

14. De la Torre, JC. Cerebral hyperfusion, capillary degeneration, and developmen of Alzheimer's disease. Alzheimer Dis Assoc Disord. 2000; 14: 572-81.

15. Giaccone G. y col, bPP and Tau interaction: A possible link between amyloid and neurofibrillary tangles in Alzheimer's disease. Am I Pathology; 1996. 148: 79-87.

16. Martínez-Miranda, A. Manual de procedimientos de enfermería sobre terapia física, musicoterapia y socioterapia para la atención del pociente con demencia tipo Alzheimer. Tesis Lic. Escuela Nacional de Enfermería y Obstetricia, UNAM, 2000. $88 \mathrm{pp}$

17. Larios-Saldaña, MA. Reflexiones sobre la multi e interdisciplinariedad en Enfermería. Enfermería Universitaria. 2004; ! (2): 29-31

\section{DIRECCIÓN PARA CORRESPONDENCIA}

Biól. Victor valverde Molina: vvalverde@eneo.unam.mx. 